\title{
Questes
}

Revue pluridisciplinaire d'études médiévales

$42 \mid 2021$

Fama : réputation et renommée

\section{Geoffrey Chaucer et le dédale de Renommée}

\section{Jonathan Fruoco}

\section{(2) OpenEdition}

\section{Journals}

Édition électronique

URL : https://journals.openedition.org/questes/5656

DOI : $10.4000 /$ questes. 5656

ISSN : 2109-9472

\section{Éditeur}

Les Amis de Questes

\section{Édition imprimée}

Date de publication : 28 janvier 2021

Pagination : 21-33

ISSN : 2102-7188

\section{Référence électronique}

Jonathan Fruoco, «Geoffrey Chaucer et le dédale de Renommée», Questes [En ligne], 42 | 2021, mis en ligne le 28 février 2021, consulté le 05 février 2022. URL : http://journals.openedition.org/questes/ 5656 ; DOI : https://doi.org/10.4000/questes.5656 


\title{
Geoffrey Chaucer et le dédale de Renommée
}

\author{
Jonathan FRUOCO \\ Université Grenoble Alpes
}

S'il existe un poète dont l'œuvre même reflète la multiplicité de sens de la fama, c'est bien Geoffrey Chaucer. En effet, selon les époques de sa vie, Chaucer semble alterner entre un désir de reconnaissance et une mise en scène de la renommée souvent très acerbe. Nous tenterons donc de questionner ici toute l'ambiguïté de la relation de Chaucer avec la notion de fama.

Bien que soucieux du devenir de son œuvre, Chaucer n'en garde pas moins une position ironique, jouant avec le topos de modestie et l'autodérision pour couvrir ses traces au sein du palais de Renommée.

\section{Chaucer à la cour : écuyer ou poète ?}

L'importance de Chaucer dans l'histoire de la littérature anglaise n'est plus à prouver : elle est admise et commentée depuis des siècles et tous s'accordent pour lui attribuer le titre de père de la langue anglaise.

Ses admirateurs, dès le $\mathrm{XV}^{\mathrm{e}}$ siècle, vont facilement s'imaginer Chaucer comme l'une des figures de proue des cours d'Édouard III et de Richard II. Pourtant, les archives nous montrent que le poète n'était pas des plus intégrés à la cour. En effet, les 493 documents officiels formant les Chaucer Life-Records ${ }^{1}$ ne font en aucun cas mention de sa carrière de poète et présentent plutôt un serviteur cherchant l'avancement auquel il ne peut prétendre par la naissance au moyen de

\footnotetext{
${ }^{1}$ Chaucer Life-Records, dir. Martin M. Crow et Clair C. Olson, Oxford, Oxford University Press, 1966.
} 
services rendus au roi et à ses alliés ou grâce à l'aide de ses proches. L'un des principaux soutiens de Chaucer à la cour est sans conteste sa femme Philippa, suivante de la reine. Chaucer lui-même entre au service de la famille royale en 1367 en tant que valettus, mais devient la même année écuyer. Cela dit, en épousant la fille du sieur Paon de Roet, chevalier de Hainaut arrivé en Angleterre avec la Reine Philippa, épouse d'Édouard III, Chaucer s'élève de manière considérable sur l'échelle sociale. Katherine, sœur de Philippa Chaucer, rend également de grands services à leur famille en entrant dans la maison de Jean de Gand en 1365, duc de Lancastre et père du futur Henri IV. Elle devient gouvernante en 1368 et prend en charge les enfants de Blanche de Lancastre et de Jean de Gand à la mort de leur mère ${ }^{2}$. Dès 1372, elle devient la maîtresse de Jean de Gand, qui s'affiche ouvertement en sa compagnie et finit par l'épouser en 1396. Bien que profitable pour Chaucer et Philippa, la relation de Katherine avec le Duc n'est pas toujours facile à gérer pour Chaucer dans la mesure où sa belle-sœur est particulièrement impopulaire dans le royaume.

Chaucer reste malgré tout en marge de la cour comme des dizaines d'autres serviteurs de rang inférieur et devient même, une fois nommé contrôleur des douanes en 1374, petit fonctionnaire. Sa carrière poétique se développe donc dans l'ombre de sa fonction officielle : en véritable Janus, Chaucer présente au monde un double visage, l'un secret, l'autre public, une dualité qui le suivra dans son approche de la fama. Le cercle littéraire de Chaucer est en effet constitué de ses proches et de quelques amis ; il semble que personne à la cour ne sait ce qu'il fait de son temps libre. L'Angleterre ne vénère ni n'adoube le poète, au contraire de la société civile florentine avec laquelle Chaucer prend contact plus tard lors de ses voyages diplomatiques. À cette époque, la société anglaise est, au contraire, encore très marquée par un univers chevaleresque où les faits d'armes comptent plus que de belles rimes. Les voisins européens de Chaucer, particulièrement en

\footnotetext{
${ }^{2}$ Paul Strohm, Chaucer's Tale : 1386 and the Road to Canterbury, New York, Viking Adult, 2014, p. 28.
} 
Italie, ne voient aucun mal à la quête de célébrité - Pétrarque (1304-1374), célébrité en son temps, étant même élevé au rang de poète lauréat à Rome. Mais l'Angleterre n'est pas encore capable de suivre les pays latins sur la voie de la fama, comme le suggère Paul Strohm, à cause de la répugnance des poètes anglais à cultiver leur propre gloire littéraire ${ }^{3}$. Si nous ignorons qui a composé Sire Gauvain et le Chevalier Vert, c'est précisément parce que son poète, comme beaucoup d'autres à cette époque, ne mentionne pas son nom d'une façon ou d'une autre dans son œuvre. Le fait d'écrire en vernaculaire accentue encore la distance que ces artistes mettent entre eux et les auteurs classiques. Ces derniers méritent le titre d'auctor, de créateurs composant des œuvres originales, là où les contemporains de Chaucer ne se voient que comme des continuateurs ou des traducteurs. Chaucer lui-même se présente comme un «lewd compilator ${ }^{4}$ ». Il fait d'ailleurs usage dans une grande partie de son œuvre d'un topos de modestie masquant son mépris pour ces poètes en quête de reconnaissance. Chaucer perçoit alors toute l'ambiguitté de la notion de fama, cette déesse à double face qui, « d'une base commune fondée sur le bruit, glisse soit vers la rumeur, soit vers la réputation ${ }^{5} »$.

C'est précisément ce qu'il illustre dans The House of Fame, premier poème composé après sa découverte de la poésie de Dante durant son passage à Florence en 1373.

\section{La Rumeur est une déesse}

La lecture de la Divine Comédie (1308-1321) fut de bien des façons une révolution aussi bien pour Chaucer que pour l'Angleterre elle-même, qui fit à cet

\footnotetext{
${ }^{3}$ Paul, Strohm, Chaucer's Tale..., op. cit., p. 204.

${ }^{4}$ A Treatise on the Astrolabe, 1. 61. Nous traduisons : « un modeste compilateur ». Toutes les citations de l'œuvre de Chaucer sont tirées de The Riverside Chaucer, dir. Larry Benson et F. N. Robinson, Boston, Houghton Mifflin, 1987.

${ }^{5}$ Claude Gauvard, «La Fama, une parole fondatrice», Médiévales, vol. 24, 1993, p. 5-13, cit. p. 5.
} 
instant ses premiers pas vers la Renaissance. Mais aussi important que put être Dante pour Chaucer, les deux auteurs ne partageaient pas, sur le fond, la même conception de la poésie. L'un est le poète de la béatitude, le père vénéré de la poésie italienne, l'autre est un homme bien plus terre-à-terre, préférant le tumulte de la place du marché à la contemplation théologique. Dante contribue dans la Comédie au développement de sa propre légende, et c'est de ce besoin de reconnaissance que Chaucer se moque éperdument dans son propre texte. The House of Fame nous invite ainsi à suivre le narrateur, nommé Geffrey, au sein d'une vision onirique : l'avatar du poète se réveille dans le temple de Vénus et fait face à des peintures murales mettant en scène l'Énéide. Sortant du temple, il réalise qu'il est perdu, et c'est alors qu'un aigle, tiré tout droit de la Divine Comédie, surgit et l'emporte vers le palais de Renommée. Chaucer se permet alors une analyse cinglante aussi bien de la vanité de ses homologues européens que des limites même de l'esprit humain et de son implication dans le processus créatif. L'aigle qui vient extirper Geffrey de sa contemplation dans le Livre II de Fame est sans conteste le motif le plus significatif emprunté par Chaucer à l'auteur italien. Dante et Geffrey sont tous deux témoins de l'arrivée de cet aigle aux plumes d'or ${ }^{6}$, fondant sur eux comme la foudre ${ }^{7}$, et l'un comme l'autre ne peuvent s'empêcher de faire référence au mythe de Ganymède ${ }^{8}$. Or si l'aigle reste apparemment en tout point similaire à son équivalent dantesque, il devient évident dès sa prise de parole que Chaucer ne compte pas imiter fidèlement le poète toscan. Alors que l'envol de l'aigle avait une grande valeur théologique et philosophique chez Dante, menant le narrateur vers Dieu et contribuant à son élévation spirituelle, il sert systématiquement à Chaucer à présenter une contrepartie ironique de l'attitude de Dante. Face à l'élévation, Chaucer opte pour le rabaissement, un

${ }^{6}$ The House of Fame, V, v. 529-533; Purgatorio, IX, v. 19-21. Toutes les citations et références à l'œuvre de Dante sont tirées de La Divina Commedia, éd. B. Garavelli, L. Magugliani, $3^{\mathrm{e} e ́ d ., ~ M i l a n o, ~ B u r, ~} 2008$.

${ }^{7}$ Fame, V, v. 534-540 ; Purgatorio, IX, v. 28-30.

${ }^{8}$ Fame, V, v. 588-591; Purgatorio, IX, v. 22-24. 
principe artistique au cœur même du réalisme grotesque et de l'esprit carnavalesque. Lorsque l'aigle emporte Geoffrey, il lui fait ainsi remarquer son aspect ventripotent, déclarant en tentant de le réconforter " "Seynte Marye, /Thou art noyous for to carye!"” ", avant d'enchaîner avec ce que David Wallace décrit comme un commentaire audio touristique particulièrement fastidieux ${ }^{10}$. De même, là où Dante se demandait s'il était digne de suivre Virgile dans ce voyage, précisant qu'il n'est ni Énée, ni Paul ${ }^{11}$, Geffrey s'interroge : « Wher Joves wol me stellyfye, /Or what thing may this sygnifye? II neyther am Ennok, ne Elye ${ }^{12} »$.

L'aigle a bien évidemment son mot à dire sur la question et rétorque assez sèchement "Thow demest of thyself amys ${ }^{13}$ ». Jupiter n'a en aucun cas d'aussi hauts desseins en vue pour le pauvre Geffrey. Le narrateur se demande même s'il est présent dans ce rêve ou s'il s'agit d'une projection de son âme, une interrogation récurrente chez Dante ${ }^{14}$, un doute que l'aigle interrompt une nouvelle fois en s'écriant «Lat be [...] thy fantasye $!^{15} »$. On le voit, Chaucer adopte une attitude ouvertement ironique et moqueuse vis-à-vis de Dante. Il ne partage pas l'intérêt du Florentin pour la célébrité et fait preuve d'un certain mépris qu'il illustre une nouvelle fois avec son ironie habituelle, ce qui exacerbe par contraste sa propre modestie. Arrivé enfin devant le palais de Renommée, il s'aperçoit que le piton escarpé sur lequel est bâti l'édifice est fait d'un matériau des plus instables, puisque la roche laisse place à des fondations de glace. Décrivant la fragilité de ces fondations, Geffrey songe alors que le constructeur a bien peu de raisons de se vanter ${ }^{16}$ ! La glace, couverte de noms de personnalités,

\footnotetext{
${ }^{9}$ II, v. 572-574. Nous traduisons : « Oh, Sainte Marie, tu n'es pas commode à porter !».

${ }^{10}$ David Wallace, «Chaucer's Continental Inheritance : the Early Poems and Troilus and Criseyde », dans The Cambridge Chaucer Companion, dir. Piero Boitani et Jill Mann, Cambridge, Cambridge University Press, 1986, p. 19-37, cit. p. 24.

${ }^{11}$ Inferno, II, v. 32.

${ }^{12}$ Fame, II, v. 586-588. Nous traduisons : «Jupiter veut-il me changer en astre ? /Qu'est-ce que cela peut-il signifier? /Je ne suis point Énoch, ni Élie ».

${ }^{13}$ Fame, II, v. 596. Nous traduisons : « Tu te trompes à ton sujet».

${ }^{14}$ Voir notamment Paradiso, I, v. 73-74.

${ }^{15}$ Fame, v. 992. Nous traduisons : «Allons donc, cesse de rêver! ».

${ }^{16}$ Fame, III, v. $1128-1135$.
} 
commence même à fondre par endroits, effaçant au passage les inscriptions dont elle est couverte.

Bien décidé à en voir plus, Geffrey continue néanmoins à avancer et arrive bientôt devant le palais.

L'attitude de Chaucer vis-à-vis de la transmission de son œuvre est à l'image de sa conception de la célébrité, multiple et changeante. Au XIV siècle, la poésie est à un moment crucial de son développement, tiraillée entre oralité et diffusion écrite. La poésie de Chaucer, comme celle de ses contemporains, est alors certes lue en public mais non pas pour autant improvisée lors de la performance ; il s'agit de poèmes composés dans l'intimité du poète, écrits sur des tablettes ou manuscrits et récités ensuite devant un auditoire ${ }^{17}$. S'il n'y a pas de circulation des manuscrits, les textes, eux, se diffusent à la faveur des lectures publiques. Chaucer illustre cette dualité dans sa description du palais de Renommée. L'extérieur est ainsi consacré à la culture orale : Geoffrey y voit notamment Orphée jouant de la harpe avec Orion, Chiron et d'autres harpistes. Il aperçoit des milliers de ménestrels, musiciens et voyants ${ }^{18}$, ainsi qu'un si grand nombre d'autres artistes de rue qu'il pourrait, dit-il, nous en faire la liste toute la journée durant. L'intérieur du palais, en revanche, est dédié à la littérature écrite. Chaucer nous rappelle ici toute la difficulté qu'il y a, à son époque, à séparer l'écrit et l'oral : il va donner une voix aux poètes dont la notoriété est si grande qu'elle leur vaut de devenir les piliers de la voûte du palais. Cependant, cette renommée n'est pas suffisante à leurs yeux, puisqu'ils continuent de rivaliser les uns avec les autres : Geoffrey entend les auteurs classiques ayant immortalisé la guerre de Troie se disputer, certains accusant par exemple Homère d'avoir favorisé les Grecs dans sa version du récit ${ }^{19}$. En dépit de leur gloire, tous ces artistes restent condamnés, comme autant d'âmes destinées à errer dans les limbes,

\footnotetext{
${ }^{17}$ Paul Strohm, Chaucer's Tale..., op. cit., p. 190

${ }^{18}$ Fame, III, v. 1201-1281.

${ }^{19}$ Fame, III, v. 1477-1480.
} 
à passer l'éternité en conflit et en concurrence les uns avec les autres. On comprend alors pourquoi Geoffrey s'empresse de préciser, lorsqu'un esprit l'interroge sur ce qu'il désire, qu'il n'est pas en quête de renommée, et préfère «[t]hat no wight have my name in honde ${ }^{20} »$ après sa mort. Il est conscient de sa propre valeur et n'a besoin de l'admiration de personne. Il est loin d'avoir les mêmes prétentions que Dante et nous le fait savoir ouvertement. Là où le Florentin priait Apollon d'être digne de ses lauriers ${ }^{21}$, Geoffrey précise, non sans autodérision, qu'il se contentera d'embrasser l'arbre d'Apollon ${ }^{22}$. Quoique parodiques, ces déclarations montrent le manque d'intérêt de Chaucer pour la célébrité. Ce contraste entre l'écrit et l'oral vient appuyer l'opposition entre le public et le privé, et cristallise l'opposition entre fama et rumor. Gauvard précise à ce sujet que :

Le poids de la tradition est tel dans les périodes concernées que l'oralité l'emporte, y compris, comme le montre Paul Zumthor, jusqu'au plus profond de l'écriture où s'entend la voix. Cette société, dans son plus grand nombre, ne vit que par la parole proférée et se trouve, du même coup, sous la menace constante de subir des informations qu'elle ne peut pas contrôler ${ }^{23}$.

Or Chaucer montre une compréhension très fine de cette problématique. Se souvenant que la Rumeur est elle aussi une déesse, comme le rappelait Hésiode dans Les Travaux et les Jours ${ }^{24}$, il nous guide, par l'intermédiaire de sa persona poétique, hors du palais de Renommée et vers celui des Rumeurs. Ce dernier s'avère alors être l'antichambre de Renommée. Geoffrey découvre un palais bien différent : c'est un labyrinthe construit en osier, en rotation constante, dont les courants d'air produisent un sifflement qui n'est pas sans rappeler celui d'une

\footnotetext{
${ }^{20}$ Fame, III, v. 1877. Nous traduisons : «Que l'on oublie jusqu'à mon nom ».

${ }^{21}$ Paradiso, I, v. 13-18.

${ }^{22}$ Fame, III, v. 1091-1108.

${ }^{23}$ Claude Gauvard, « La Fama, une parole fondatrice », art. cit., p. 7-8.

${ }^{24}$ Hésiode, Théogonie ; Les Travaux et les Jours ; Le Bouclier, éd. Paul Mazon, Paris, Les Belles Lettres, 1972, p. 764.
} 
pierre lancée par une catapulte ${ }^{25}$. Les nouvelles, ou «tydynges », allant et venant du palais des Rumeurs à celui de Renommée sont essentiellement des créations verbales. L'aigle avait ainsi expliqué à Geffrey que le son «ys noght but eyr ybroken $^{26} »$ : les paroles prononcées sont de l'air compressé s'élevant par un processus de réverbération vers le palais des Rumeurs avant de rejoindre celui de Renommée. Or Chaucer en décrivant le son comme de l'air « brisé », c'est-à-dire une forme de flatulence, nous laisse une nouvelle fois entendre ce qu'il pense vraiment de la question! Les rumeurs ainsi personnifiées restent libres, une fois sur place, de se déplacer selon leur convenance dans cette toupie d'osier, mais rares sont celles parvenant à quitter les lieux sous la même forme qu'à leur arrivée. Vérité et mensonge peuvent se retrouver coincés l'une par l'autre près d'une ouverture, et n'ont d'autre solution pour quitter le palais que de faire un compromis :

We wil medle us ech with other,

That no man, be they never so wrothe,

Shal han on [of us] two, but bothe

At ones, al besyde his leve,

Come we a-morwe or on eve,

Be we cried or stille yrouned ${ }^{27}$.

Si des nouvelles mêlant le vrai et le faux parviennent à atteindre le palais de Renommée, rien ne prouve dès lors que cette dernière n'a pas accordé sa grâce à des nouvelles en partie mensongères. À qui sait écouter, écrit Marcel Detienne, toute rumeur fait signe, mais c'est alors une voix ponctuelle, instantanée et qui est comme un atome de la Rumeur constituée, de celle qui, relayée de bouche en bouche et d'oreille en oreille, se métamorphose en récit déjà formel, chacun y ajoutant ou en retirant quelque

\footnotetext{
${ }^{25}$ Fame, III, v. 1916-1955.

${ }^{26}$ Fame, II, v. 765. Nous traduisons : « n'est autre que de l'air brisé ».

${ }^{27}$ Fame, III, v. 2102-2107. Nous traduisons : « Nous allons tant nous mêler, /Que nul homme, même en colère, /Ne pourra trouver l'un sans l'autre, /Il ne pourra voir que les deux, /Que ce soit à l'aube ou de nuit, /Que notre voix soit forte ou basse ».
} 
chose, par une procédure inconsciente mais toujours dans une création collective ${ }^{28}$.

Ces nouvelles, subtil mélange de vrai et de faux, se déplaçant à la vitesse du son, cette fama à la fois réputation et rumeur, sont d'autant plus nocives pour Chaucer qu'elles se font mémoire d'une renommée pour la postérité. Or les poètes, jongleurs et musiciens que Geffrey aperçoit à l'entrée du palais et « qui détiennent le verbe, sont, par excellence, les porteurs de cette mémoire qu'ils constituent et qu'ils font grandir jusqu'à la glorification ${ }^{29}{ }^{2}$.

Chaucer ne cache ainsi pas son aversion doublée d'admiration pour le magnum opus de Dante. Le tempérament du poète est contraire à cette quête d'autopromotion et tout nous laisse imaginer un homme discret et privé ${ }^{30}$, écrivant pour son plaisir et celui d'un cercle restreint d'initiés plutôt que pour un large public constitué d'inconnus qui liraient son œuvre sans sa supervision. Toutefois, le contexte culturel et scientifique de l'époque évolua et le contraignit à revisiter ses prises de position. Sa décision d'écrire en moyen-anglais durant les années 1360 eut en effet plus d'impact qu'il ne l'avait pensé et d'autres, tels que son ami John Gower, l'imitèrent. Les évolutions technologiques permirent en outre de faire circuler plus aisément les manuscrits.

Durant la deuxième moitié du siècle, ses contemporains commencèrent à s'attribuer la paternité de leurs œuvres et la composition de Troilus and Criseyde en 1385-1386 prouve que Chaucer lui-même, en dépit de doutes toujours présents,

\footnotetext{
${ }^{28}$ Marcel Detienne, «La Rumeur, elle aussi, est une déesse », Le Genre humain, vol. 5, 1982, p. 71-80, cit. p. 77.

${ }^{29}$ Claude Gauvard, «La Fama, une parole fondatrice », art. cit., p. 10.

${ }^{30}$ L'aigle de The House of Fame lui reproche ce tempérament, précisant qu'il ne sait rien sur les joies du peuple d'Amour, ni sur les êtres créés par Dieu, qu'ils soient lointains ou ses propres voisins, à quelques mètres de sa porte : "For when thy labour doon al ys, /And hast mad alle thy rekenynges, /In stede of reste and newe thynges /Thou goost hom to thys hous anoon, /And, also domb as any stoon, /Thou sittest at another book /Tyl fully daswed ys thy look ; /And lyvest thus as an heremyte » (II, v. 652-659).

Nous traduisons : "Car une fois ton travail accompli /Et tes comptes bien faits, /Plutôt que repos et nouveautés, /Tu rentres chez toi aussitôt /Et alors, muet comme une pierre, /Tu t'assois avec un autre livre/Jusqu'à en être étourdi ; /Tu vis ainsi comme un ermite ».
} 
faisait désormais preuve d'une ambition littéraire nouvelle et qui aurait fortement surpris le Geoffrey de The House of Fame.

\section{L'appel de Renommée}

En traduisant Il Filostrato (1335-1340) de Boccace en anglais, Chaucer n'avait sans doute pas songé qu'il livrerait à la littérature anglaise l'une de ses œuvres fondatrices. Il semble pourtant qu'une fois le poème achevé, il se retrouva face à une situation nouvelle. Comme le remarque Strohm,

Chaucer responds in an agitated way to an idea that appears to have just occurred to him, or that is striking him with unprecedented force: He has written a poem deserving of broad admiration, a poem that may circulate to present and future audiences in manuscript form, and thus be read without his superintending presence and beyond his personal control ${ }^{31}$.

Par sa nouveauté, cette attitude laisse transparaître toute l'ambiguïté de Chaucer qui effleure l'idée de diffuser son œuvre tout en restant méfiant. Il n'avait jusqu'alors eu à faire qu'à un public de taille modeste, et dont la réception de l'œuvre était contrôlée et dictée par le poète lui-même lors de la lecture. Or diffuser son œuvre implique de perdre ce contrôle, tant sur la réception que sur la production même des manuscrits. Certes, Chaucer n'est plus aussi absolu vis-àvis de la fama, mais il ne souhaite aucunement que le renom auquel il pourrait désormais avoir droit soit conditionné par une falsification du réel résultant d'une mauvaise diffusion de l'information.

Il parvient toutefois dans la conclusion de Troilus and Criseyde à laisser partir, presque à contrecœur, son « litel bok $k^{32}$ », ce petit livre auquel il tient tant. S'il estimait autrefois ne pas être digne des lauriers d'Apollon, il s'inscrit

\footnotetext{
${ }^{31}$ Paul Strohm, Chaucer's Tale..., op. cit., p. 209.

${ }^{32}$ Le fait même que Chaucer fasse désormais référence à son œuvre comme à un livre, «bok», est une preuve supplémentaire du changement de l'état d'esprit du poète et de la matérialité de l'œuvre.
} 
désormais ouvertement dans une tradition poétique remontant aux grands classiques de 1'Antiquité :

But litel book, no making thou n'envye,

But subject be to alle poesye ;

And kis the steppes wher as thow seest pace

Virgile, Ovide, Omer, Lucan, and Stace ${ }^{33}$.

Ne pouvant plus suivre ses vers, il les envoie chercher leur propre fortune dans le palais de Renommée. Il ne prend pas le titre d'auctor, mais se présente pourtant comme héritier de ces grands noms. Toujours inquiet, en dépit de ses ambitions auctoriales, au sujet d'une possible mauvaise circulation des manuscrits ${ }^{34}$, il prie pour que ses vers soient bien compris et ne soient pas déformés ou pervertis lors de leur transmission. Ce n'est d'ailleurs pas la première fois que Chaucer s'inquiète ouvertement de l'état de ses manuscrits. Dans un court poème intitulé «Chaucers Wordes unto Adam, His Owne Scriveyn», il réprimande son copiste, Adam Pinkhurst, pour son manque de rigueur dans ses copies de Troilus et de la traduction par Chaucer de La Consolation de Philosophie de Boèce. Il écrit que la négligence d'Adam le force à corriger ses copies et qu'il doit à chaque fois reprendre son travail ${ }^{35}$. Cette petite leçon poétique donnée à son copiste prouve que Chaucer était déjà capable de mettre une certaine distance entre son activité de poète et celle d'un simple employé copiste.

Les ambitions de Chaucer se précisent jusqu'à la fin de sa carrière et l'on voit dans chaque nouvelle œuvre une affirmation de ses prétentions aux lauriers d'Apollon. Que ce soit par choix ou par nécessité, comme lors des changements

${ }^{33}$ Fame, V, v. 1789-1792. «Garde-toi, petit livre, d'être jaloux, /Incline-toi devant vraie poésie ; /Et baise la trace qu'ont laissée de leurs pas /Virgile, Ovide, Homère, Lucain et Stace ». ${ }^{34}$ Fame, V, v. 1793-1798.

${ }^{35}$ Voir le court poème Chaucers Wordes Unto Adam, His Owne Scriveyn, dans lequel il écrit : « So ofte adaye I mot thy werk renewe, /It to correcte and eke to rubbe and scrape, /And al is thorugh thy negligence and rape ».

Nous traduisons : « Souvent chaque jour je dois te reprendre, /Te corriger et gratter et frotter, /Et tout cela par ta négligence et hâte ». 
dramatiques de $1386^{36}$, Chaucer innove de plus en plus et développe considérablement, dans ses deux dernières œuvres, la portée de ses vers. Il fait par exemple à deux reprises, dans The Legend of Good Women, une liste de ses compositions, cherchant peut-être à préserver pour la postérité sa bibliographie complète. Et bien qu'inachevés et édités par Adam Pinkhurst, ses Contes de Canterbury marquent un véritable tournant, tant dans sa conception de la littérature que dans sa relation avec le public présent et futur. Pensant cette fois véritablement son œuvre comme un livre, il n'hésite plus à encourager le lecteur à tourner la page et choisir un autre conte pour ne pas avoir à faire face à la vulgarité d'un des pèlerins ${ }^{37}$.

La mort de Chaucer en 1400 l'empêcha d'être témoin de la diffusion de son œuvre. Le changement de régime politique qui venait alors d'avoir lieu mit sur le trône d'Angleterre une nouvelle dynastie. Henri IV et la faction lancastrienne se montrèrent bien plus encourageants que Richard II en ce qui concerne la production littéraire en vernaculaire. Les changements culturels et sociétaux observables dans les années 1380 atteignirent leur apogée durant ce nouveau siècle. À la mort de John Gower, huit ans après celle de Chaucer, sa tombe fut recouverte d'un somptueux monument sur lequel on peut toujours lire « Hic jacet J. Gower Arm. Angl. Poeta celeberrimus ${ }^{38} »$. Une telle reconnaissance et renommée aurait été impensable quelques décennies plus tôt et prouve non seulement l'infléchissement de la politique anglaise vers une sacralisation de sa langue, mais également l'évolution des mentalités des auteurs eux-mêmes.

Enterré en toute discrétion au sein de l'abbaye de Westminster, Chaucer n'eut pas droit aux éloges de Gower. Sa sépulture fut la première à occuper le

\footnotetext{
${ }^{36}$ L'année 1386 fut particulièrement difficile pour Chaucer puisqu'il perdit son emploi de contrôleur des douanes à Londres. Il se retrouva du jour au lendemain sans logement, sans revenus et, étant obligé de quitter la ville, privé de son public.

${ }^{37}$ The Miller's Prologue, v. 3176-3177.

38 « Gower, Esq. : très célébré poète de l'Angleterre ». Le titre « esquire » conféré à Gower est traditionnellement l'équivalent du rang d'écuyer, mais sert surtout de titre de respect afin de noter le statut social d'une personne.
} 
Coin des Poètes de l'abbaye, qui accueillit par la suite, entre autres, les restes d'Edmund Spenser, d'Alfred Lord Tennyson, de John Dryden et de Charles Dickens. Après avoir longtemps fui les honneurs de Renommée et avoir erré dans son dédale, Chaucer devint finalement malgré lui l'architecte de son propre palais. 
